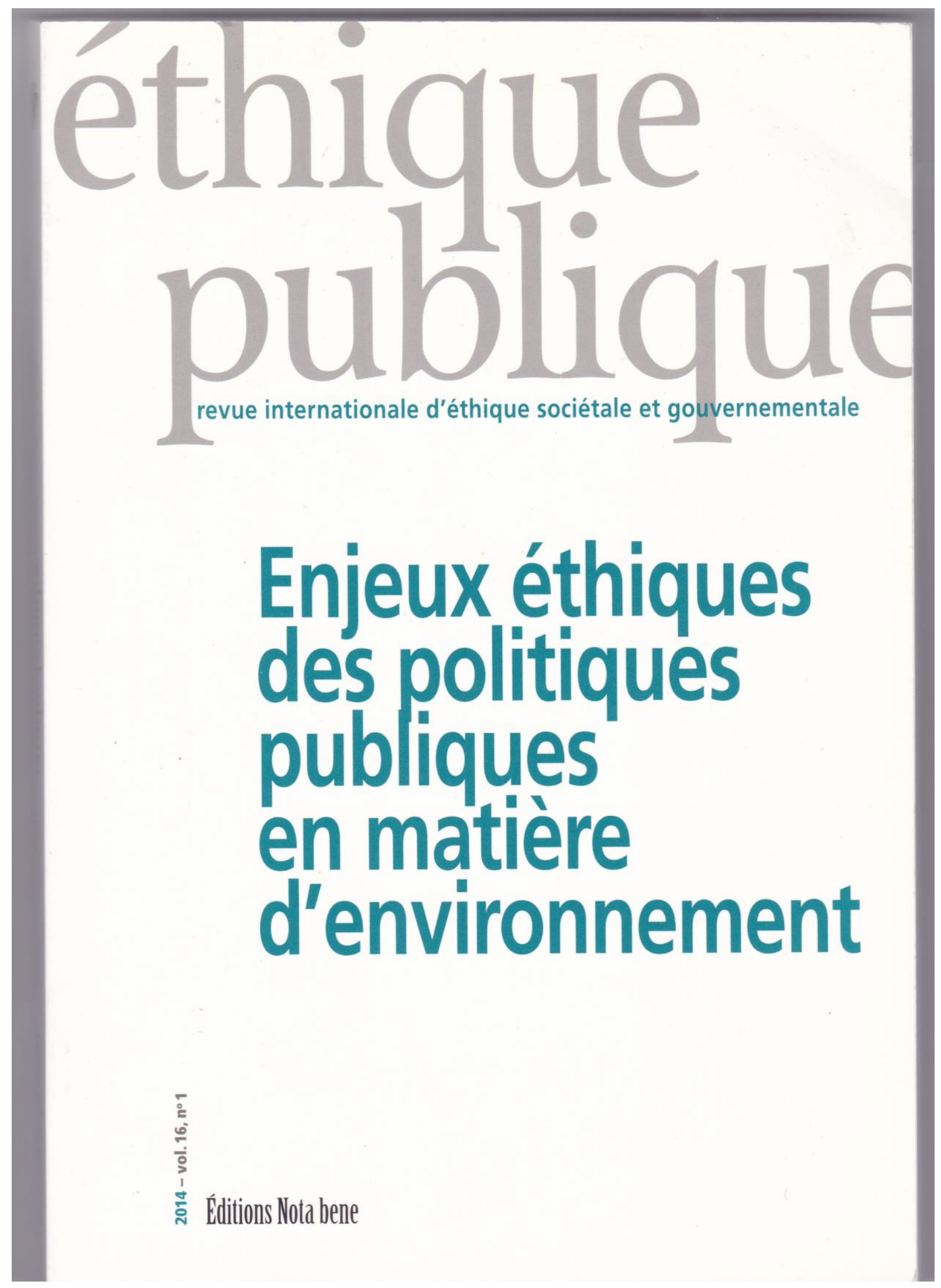




\section{Vers une dictature de la transparence : secret et démocratie}

\section{Introduction}

La présente réflexion est issue d'une recherche intitulée «Secret militaire et participation », qui fut menée de 2010 à 2013 par une équipe pluridisciplinaire à laquelle j'appartins dans le cadre du programme de recherche du Ministère de l'Ecologie baptisé «Concertation, décision, environnement ». Elle en constitue plus précisément le fondement théorique et tente de mettre en relation les mutations du pouvoir à l'œuvre depuis les trente dernières années et les enjeux démocratiques liés aux sociétés du risque à travers une question éthique essentielle: le statut du secret dans les démocraties. Nous pouvons dire qu'il s'agit d'une question éthique au sens où la revendication d'un droit à la connaissance est devenue une question politique fondamentale au sein des sociétés technoscientifiques, en particulier dans l'expertise des risques sanitaires et environnementaux. Les débats liés à la traçabilité, le choix européen d'étiqueter les OGM destinés à l'alimentation humaine, la directive REACH recensant les produits dangereux, etc., posent clairement la responsabilité morale des industriels et des institutions publiques censées garantir la sécurité des citoyens telles que les Agences sanitaires (ANSES pour la France, EFSA pour l'Europe). Dès lors la question se pose de savoir, comme c'est le cas pour les biotechnologies alimentaires, jusqu'où peut-on opposer le secret industriel au nom de la protection des brevets lorsqu'une controverse sanitaire se fait jour. Mais la dimension éthique du secret renvoie plus fondamentalement à sa nature-même, c'est-à-dire la logique de séparation qui le définit, comme nous le verrons, comme sacré. Nous aborderons donc le secret à ces deux niveaux, dans sa double dimension politique et anthropologique, la première renvoyant à la question du pouvoir et plus largement de l'administration des «affaires de la cité », et la seconde, à la dimension symbolique qui fonde toute activité humaine et par laquelle chaque société cherche à définir une humanité de l'homme. Ethique, secret, politique et anthropologie sont, au fond, autant de manières de poser la question des limites.

Si l'étude de la problématique du voilement et du dévoilement dans les rapports asymétriques du pouvoir est une tradition déjà ancienne en philosophie politique, l'étude sociologique du secret n'a été inaugurée par Simmel qu'au début du XXe siècle, lequel en étudia l'effet sur les formes sociales. Le secret fut conçu en sociologie dans son acception la plus générale comme «limitation de la connaissance réciproque » (Simmel, 1991) sous la forme de quatre figures principales que sont le non-dit, le mensonge, l'intime et la connivence. Il est étonnant qu'il soit aujourd'hui si peu étudié en tant qu'objet propre, notamment dans le cadre du débat public sur les risques, comme si la modernité avait, sous l'éclairage des Lumières, réduit à néant toutes les zones d'ombre qui obscurcissaient les sociétés traditionnelles. A. Petitat (1998), qui a repris le questionnement de Simmel, nous propose un éventail élargi de catégories mettant en jeu simultanément plusieurs formes du 
voilement/dévoilement : le mensonge illicite (tromperie, imposture, diffamation), le mensonge licite, le non-dit illicite (non-dit mensonger ou trompeur, censure), le non-dit licite (privé, discrétion), l'expression non déformante illicite (dévoilement brutal), l'expression non déformante licite (aveu, authenticité). La combinaison de ces postures de base permet de retracer un grand nombre de figures interactives marquées par le secret et la révélation, auxquelles n'échappent pas les modalités de la concertation autour des questions environnementales, surtout lorsqu'elles se heurtent au secret défense.

Le secret défense, qui constituera l'objet de départ de notre réflexion, mais pas le seul, est de ce point de vue un mélange de non-dit licite, de mensonge et de tromperie. Il constitue aussi, comme nous allons le voir, un révélateur qui, tout en dissimulant, dévoile sa propre nature. Toute dissimulation, à partir du moment où elle s'explicite (volontairement ou non) n'en dit-elle pas autant sur celui qui voile que sur ce qui est caché ? Comment, dès lors, comprendre la coexistence, voire l'articulation des actuelles logiques de «transparence » et de dissimulation à l'œuvre dans nos sociétés? Qu'il s'agisse des questions d'Etat, de l'évaluation technoscientifique ou de moralité de la vie politique, une même question semble se poser : quelle est la légitimité du secret et jusqu'où peut-on, doit-on le tenir ? Quelle en sont, enfin, les enjeux politiques pour la démocratie?

\section{Concertation et secret}

Tantôt les institutions politiques s'entourent d'une épaisse opacité, tantôt elles se tournent vers un idéal de transparence, en particulier les institutions démocratiques. Georg Simmel remarquait, il y a plus d'un siècle, que la société moderne occidentale est prise dans des tendances contradictoires dans la mesure où « la révolution démocratique et l'avènement d'un espace public renforcé par les mass media ont indubitablement conduit à une transparence accrue du politique »(Petitat, 1997). Mais les théories générales du secret débouchèrent plutôt dans un premier temps sur la notion d'incertitude, au cœur de l'actuel débat sur les risques industriels et technologiques territorialisés : incertitude de la connaissance réciproque, incertitude des postures interactives. L'espace incertain des interactions appelle nécessairement des mécanismes de réduction de l'incertitude qui constituent autant de formes sociales liées aux conditions de base du voilement/dévoilement symbolique : l'intériorisation des conventions, les punitions et récompenses, l'intérêt commun à la continuité des échanges, l'impossibilité du mensonge généralisé, le souci de la réputation, l'exigence de plausibilité et enfin la confiance. Cette dernière question suscite désormais un regain d'intérêt dans les crises environnementales et les débats d'experts, notamment après une longue série «d'affaires ». A qui se fier ? Qui croire ? On peut certes la définir comme un état d'ouverture affectif et cognitif tendu vers la possibilité d'un échange sans risque, contrairement à l'agone. Elle représente une sorte de «mise de départ » ou d'espoir qui soutient l'engagement dans le jeu des échanges, ici la concertation. Le secret est donc, dans l'idéal démocratique, habituellement associé à une conception négative de l'interaction alors qu'il est - paradoxalement - inhérent à la constitution de l'Etat. 
C'est la nature contradictoire des nouveaux enjeux (politiques, sociaux, technologiques et militaires), entre les obligations stratégiques liées au secret d'une part, et les exigences de transparence et diffusion de l'information portées la société d'autre part, qu'il nous a semblé pertinent d'étudier dans une perspective socio-anthropologique, entre des formes de gouvernance issues du passé (imposition du secret militaire) et la finalité nouvelle d'installations militaro-industrielles de plus en plus soumises à l'ouverture et à la concurrence, destinées à produire des innovations technologiques pour le marché international telles que l'usine de Valduc, Installation Nucléaire de Base Militaire (du CEA) destinée à la fabrication de la charge explosive des missiles nucléaires français, où nous enquêtâmes. Pour caractériser ce nouveau contexte caractérisé par un passage d'une logique de surveillance du territoire à la maîtrise des flux, nous pourrions repartir des travaux de Paul Virilio (1993) qui a tenté de saisir la complexité des appareils militaires en regard des questions de sécurité des territoires, et de ceux Michel Foucault et de Gilles Deleuze pour leur réflexion sur l'idée contrôle. Selon Foucault (1975), nous sommes entrés depuis un demi-siècle dans une crise généralisée de tous les milieux d'enfermement (prison, hôpital, usine, école, installations militaires), milieux soumis à d'incessantes réformes visant à les décloisonner et à les déterritorialiser au profit d'une gestion dynamique des flux. Les installations militaires font elles aussi partie de ces lieux clos soumis aux exigences contemporaines, et du coup contradictoires, de transparence. Les «sociétés de contrôle » (Deleuze, 1990) sont donc en train de remplacer les anciennes sociétés disciplinaires. Paul Virilio n'a cessé d'analyser à son tour les formes ultra-rapides de contrôle à l'air libre, qui remplacent les vieilles disciplines opérant dans la durée dans un système clos. De ce point de vue, la nature du secret militaire, qui occupe une double fonction symbolique (instituer un monde littéralement « sacré », c'està-dire séparé, coupé du reste de la société) et pratique (stratégique), est devenu de plus en plus problématique. La pollution industrielle à l'extérieur d'un site tel que l'usine de Valduc par exemple, lorsqu'elle est analysée par un organisme indépendant des autorités militaires, peut apparaître aux yeux ces dernières comme une signature gênante des activités secrètes qui se déroulent à l'intérieur de l'établissement, raison qui conduit à tenter d'en interdire l'analyse, ce qui est devenu contraire à la loi, notamment la convention d'Aarhus sur l'accès à l'information, la participation du public au processus décisionnel et l'accès à la justice en matière d'environnement, signée le 25 juin 1998 par 39 États. Le déchet ou la pollution deviennent alors une information parmi d'autres, objets de voilement/dévoilement, en même temps qu'ils acquièrent une signification, au-delà du discours rationalisant sur les risques calculables. Le problème est que l'on se retrouve, au travers de cet exemple, dans une situation apparemment paradoxale, pris entre l'exigence d'information du public sur des activités dangereuses et la nécessité de tenir au secret ces mêmes activités lorsqu'elles relèvent de la défense. Il nous faut donc, à ce stade, examiner à nouveaux frais les logiques sociales et politiques du secret en reprenant notamment les apports de l'anthropologie, pour mieux en saisir l'équivocité fondamentale. Ce détour anthropologique nous semble d'autant plus nécessaire pour la formulation de pistes de réflexions pratiques permettant de dépasser l'apparente contradiction. 


\section{Retour aux sources de l'anthropologie politique : le pouvoir comme sacré.}

Selon la tradition anthropologique, notamment l'anthropologie du pouvoir telle qu'elle a pu être développée par les africanistes, par exemple chez Georges Balandier, transparence et secret sont deux notions qui s'articulent, dès que l'on tente d'approcher la question du pouvoir, plus qu'elles ne s'excluent. C'est que pour l'anthropologue, comme pour le philosophe, la «marque du sacré » (Dupuy, 2009) n'a pas disparu avec l'avènement des sociétés modernes, elle s'est éclipsée, déplacée, remodelée en fonction des normes, institutions et valeurs du moment, ces dernière n'étant jamais totalement universalisables, bien que l'on puisse s'accorder avec Serge Latouche sur le constat d'une certaine « occidentalisation du Monde » (Latouche, 2005). Pour Dupuy d'ailleurs, c'est le sacré qui nous a constitués en tant qu'humains, la désacralisation du monde nous apparaissant ainsi comme un processus inédit qui nous laisse sans protection face à notre propre violence, la vie humaine n'ayant plus de valeur (morale). Tout pouvoir peut donc être identifié comme sacré en même temps qu'il doit, pour subsister, s'appuyer sur des processus ou des logiques plus ou moins profanes de légitimation de celui-ci. Il nous faut ici insister sur la question de la légitimation, c'est-à-dire de la manière dont un pouvoir tire de la reconnaissance de la part de ceux qui en sont les sujets, dans un imaginaire social donné et à une époque donnée. Si nous entendons par légitimation le « procédé par lequel un pouvoir ou un régime politique parvient à susciter et à maintenir l'adhésion des citoyens » (Arnaud, 1993), tout état d'équilibre politique suppose que l'adhésion des citoyens découle de la conformité du pouvoir à leur imaginaire social, celui-ci pouvant être axé tantôt sur le secret (sociétés traditionnelles) tantôt sur la Raison et la Vérité (à partir des Lumières) (Bissot, 2013). Nous aurions donc, au départ de notre réflexion, deux types de pouvoir exclusifs l'un de l'autre : c'est du moins la manière dont la modernité occidentale a construit, toujours dans une perspective binaire et progressiste, sa lecture de l'histoire. C'est qu'à force de construire, d'imposer et d'enseigner le positivisme, c'est-à-dire l'idée d'une adéquation entre la vérité et l'apparence, celui-ci a fini par s'imposer, tout particulièrement dans les imaginaires contemporains façonnés par les écrans d'ordinateurs et la télévision : ce qui s'est réellement passé doit être vu et ce qui est vu ne peut s'être que réellement passé. «Le pouvoir passant par l'image est ainsi reconnu par tous les sujets du pouvoir qui s'y identifient et le considèrent dès lors comme légitime » réaffirme Hugues Bissot (ibid. p. 3). La téléréalité - ce mot prend ici une force toute conceptuelle - nous conduit à rêver d'un pouvoir direct, pur, transparent, absolu, celui d'associer le savoir au voir en dehors de toute possibilité de jeu, de faux-sembant, de tromperie, de distorsion,... qui sont pourtant le fondement du langage (et de toute la pensée psychanalytique). Peu importe que ces mêmes moyens nous révèlent la nature artéfactuelle des personnalités politiques qui s'exhibent dans leur «vérité » au petit écran, voire la nature programmatique des stratégies de communication qui président à la construction de cette « vérité » dévoilée, il faut y croire. Ou plutôt, il faut faire comme si nous y croyions. Partout d'ailleurs où la transparence est appelée (institutions politiques et institutions d'Etat, grandes entreprises, industrie nucléaire,...), partout le secret et l'obscurité se donne à voir comme seule objectivité perceptible. Lorsque Marc Abéles se penche sur l'Assemblée nationale, il remarque ainsi une certaine propension à «y entretenir certaines zones d'ombre, sans qu'on 
sache trop pourquoi » sauf à considérer «l'importance continue du secret comme essentiel à toute forme de pouvoir » (Gueshière, 2000).

Le pouvoir est pour l'anthropologie un lieu séparé, isolé, hypostasié (Clastres, 1974), coupé du corps social et qui doit en être maintenu à distance, en rupture d'échange symbolique (Moreau-de-Belaing, 2003), notamment par l'intermédiaire du secret. Le secret d'Etat, en maintenant les citoyens hors de l'Etat comme lieu du pouvoir, autorise en retour la légitimation de ce dernier, à condition toutefois que la manière dont celui-ci suscite l'adhésion et la reconnaissance soit en phase avec l'imaginaire social de la société. Il faudra donc nous arrêter plus loin sur les raisons qui semblent animer la délégitimation du secret défense, et plus particulièrement du secret militaire nucléaire. Secret et sacré font tous deux référence à la séparation, à la rupture, la pratique du secret n'est donc en rien incompatible - bien au contraire - avec l'exercice du pouvoir en général, ou d'un pouvoir en particulier. La transparence en revanche, pour atteindre à la sacralisation, doit relever un défi, celui de maintenir, en l'anéantissant, la distance qui séparait l'Etat de l'individu, ou encore le lieu du pouvoir et les citoyens. Or le pouvoir ne peut s'incarner qu'à travers différentes réalités sociales, ou encore institutions, formes ou choses qui s'imposent à ceux qui le légitiment. Dès lors, trois âges semblent baliser son histoire : la Religion (sociétés traditionnelles), le Droit (la modernité), et enfin, l'image (sociétés postmodernes ou technoscientifiques), qui constituent autant de modes de légitimation du pouvoir. Nous allons donc nous attarder sur le moment qui nous intéresse, à savoir le passage de la seconde à la troisième époque, celle des sociétés de contrôle.

\section{Secret, monde réel et ontologie}

Finalement, entre le «tout dire » et le « rien dire du tout » n'existe-t-il pas des degrés intermédiaires? Un dévoilement partiel n'est pas plus un mensonge qu'une révélation partielle, une vérité. Dire, c'est précisément élaborer une parole en direction de l'autre, une adresse à l'autre, porteuse de signification. Etre capable de garder un secret, ne pas tout dire, c'est ne pas devenir «transparent» au regard des autres et permettre de construire ainsi une relation de confiance. La transparence est la qualité de ce qui laisse passer la lumière, de ce qui n'arrête pas le regard, de ce qui finalement se laisse traverser sans apparaître. Si le secret renvoie bien à l'idée de séparation ou de sacralisation au sens anthropologique, avec la transparence disparaît la frontière, la limite et avec, la possibilité de concevoir l'intériorité et l'extériorité. Avec le dedans et le dehors, s'abîme également la nécessaire distinction opérée entre espace public et espace privé, fondement du politique. S'agissant du sujet, nul doute que transparence et dissolution de l'être - de l'être-au-monde comme de l'être ensemble - ne font qu'un, l'expérience totalitaire l'a prouvé (Arendt, 2005). Etre à l'autre et être avec les autres suppose qu'une part d'ombre abrite le lieu des secrets et de la conscience afin qu'une relation de confiance s'établisse entre les personnes, entre les groupes, entre les individus et les institutions. La transparence absolue empêche toute prise, toute consistance, et plus fondamentalement la possibilité de faire exister un monde réel (Baudrillard, 1995). Resituée dans le contexte politique, la transparence peut ainsi apparaitre comme la meilleure alliée des 
sociétés de contrôle et le pire ennemi de la démocratie, détruisant la réalité comme construction sociale (Berger, Luckman, 1986) au profit d'une virtualité insaisissable et suprapolitique. Rappelons que Berger et Luckman ont inauguré une sociologie de la connaissance alliant complexité, interactionnisme et phénoménologie, montrant que toute réalité, la réalité, est construite socialement. L'importance accordée à celle-ci ainsi qu'à la connaissance provient de leurs relativités sociales, « la réalité de la vie quotidienne se présente à moi comme un monde intersubjectif, un monde que je partage avec les autres ». La réalité est donc objective et subjective. Hugues Bissot pense fort justement, dans le sillage de note réflexion, que «si nous pensions que la transparence comme vertu suprême n'avait de secret pour personne, il était tout aussi illusoire de penser que le secret n'a pas besoin, à son tour, d'une certaine publicité et notamment qu'il s'attache au pouvoir»(Bissot, op. cit. p.4). L'auteur précise, avec R. Bertrand (cité par Bissot, ibid. p. 5), que «l'efficacité du secret comme mode de légitimation suppose que son existence soit reconnue et son contenu, ignoré ». Il nous apprend ainsi que si «pour fonder une légitimité politique, une certaine forme de commerce avec l'invisible est nécessaire et doit se donner à voir» mais que «toutes les formes de ce commerce ne sont pas bonnes à voir, à révéler, car ladite légitimité est fonction des imaginaires sociaux et de l'interprétation culturelle ». La face obscure du pouvoir fait partie de son double visage mais elle ne peut, tout en devant faire partie intégrale de celui-ci, être montrée, tout comme nous ne pouvons imaginer la lune sans sa face cachée.

Confiance, défiance, conflit et dialectique sont les dimensions essentielles du politique, comme nous l'a enseigné toute la pensée politique du XXème siècle. La visibilité absolue - sur une vérité supposée unique - est de facto vouée à l'échec. Ce fut le projet des totalitarismes (Arendt, op. cit.), régimes politique résolument modernes qui ont profondément marqué l'histoire de la modernité politique en ce qu'ils reposaient sur une volonté inédite de contrôler l'activité des hommes, non plus, comme dans la dictature, par la seule répression des oppositions, mais en tentant de s'immiscer jusque dans la sphère intime de la pensée en imposant une idéologie unique. Il s'agissait de créer en quelque sorte un système sans extériorité et qui ferait office de monde. Mais vouloir nier l'opacité fondamentale du réel «signifie non seulement neutraliser toute barrière entre les choses, mais aussi effacer les choses elles-mêmes : l'inflation du visible, par la substitution de l'espace du dehors à l'espace interne, supprime paradoxalement l'espace» (Marzano, 2009). Et l'auteur d'ajouter que «c'est seulement lorsqu'il comprend que les autres ne sont pas tout-puissants qu'un individu peut affirmer sa propre différence et sa propre autonomie ». Le maintien des ces limites, de ces barrières qui séparent le privé du public, le dedans du dehors, et qui vaut pour les individus comme pour les communautés partageant un secret, est donc la condition requise pour pouvoir vivre dans le monde réel et pouvoir construire socialement cette réalité.

\section{Le droit à l'indifférence}

Le respect de l'intimité de la personne, le droit à l'image, la liberté de conscience, l'interdiction du recoupement de fichier par la loi du 6 janvier 1978 (CNIL), et bien sûr la loi française du 17 juillet 1970 protégeant la vie privée (des écoutes téléphoniques), le secret 
médical, entre autres, ne sont rien moins que la protection du «secret » propre à chacun, la part de soi qu'il n'a pas obligation de dévoiler ou qu'il ne peut exposer au regard de l'autre, qu'il s'agisse du voisin ou de l'Etat, de l'employeur ou du fonctionnaire de police, ou plus exactement la mise au secret du domaine privé. Ainsi, si secret et sacré ont bien, nous l'avons rappelé, la même étymologie, celle de la séparation, on peut considérer que le libéralisme politique fonde l'existence en commun, la vie en société, sur la protection d'un secret propre à l'individu, en en faisant un droit inaliénable, ce que Locke appelait le droit des particuliers à «l'indifférence » des concitoyens. On ne peut s'épanouir qu'à l'abri du regard de l'autre, des autres, tel est le paradigme social qui va devenir dominant dans la second moitié du XXème siècle, alors que le paternalisme, qui plaçait l'individu sous l'autorité de l'Etat, de l'Eglise ou du patron, cède le pas à une gouvernementalité - ou gouvernement des corps - plus horizontale et plus autonome, celle d'un individu surveillé certes, mais en milieu ouvert, libre de ses mouvements. Tel est le sens profond de mai 68, manifestation visible et revendiquée par la génération montante, la «jeunesse », de cette transformation politique. S'il n'est pas certain que l'individu en soi sorti plus libre et plus émancipé dans une société en voie de bureaucratisation et de technocratisation avancée, tel était en tout cas le sens du désir collectif, un désir de secret et il n'est pas certain que cet horizon d'attente ait disparu. Chacun devient, dans ce nouveau libéralisme, maître de son destin et de sa vie (choix de formation, choix du partenaire, etc.) et ces libertés s'accompagnent d'un prix non négligeable : chacun devient aussi plus responsable de ses échec face à cette injonction à l'autonomie comme l'a fort bien théorisé Erhenberg (1998). Toqueville (1981) nous avait mis en garde contre le despotisme qui menace, de l'intérieur, les démocraties, notamment lorsque la passion de l'égalité l'emporte sur le désir de liberté et dans son sillage, nombreux sont les théoriciens qui, comme Hannah Arendt ou Anthony Giddens (1994), auront poursuivi cette mise en garde, décelant au sein même des sociétés prônant l'autonomie de l'individu des mécanismes subtils et souvent sournois, tendant à le replacer sous le contrôle de l'Etat.

\section{Crise de confiance et risque}

C'est dans les années 1990 que l'on commence à parler de «transparence », celle des institutions bien entendu, dans le sillage du mouvement amorcé une décennie plus tôt : les citoyens doivent être tenus informés de ce qui les « regarde » et les concerne, c'est-à-dire des affaires de l'Etat. L'avènement de la société du risque, autoréflexive au sens de U. Beck (2001) et plus largement la réflexion engagée par les sciences humaine sur la participation citoyenne aux débats publics, est une marque très caractéristique de l'exigence d'un droit à l'information, d'un droit de savoir et de connaître ce qui nous menace en l'occurrence. Le contexte des « affaires » et des «scandales », c'est-à-dire toutes les situations de crises qui ont maillé les décennies 80 et 90 telle que le sang contaminé, la «vache folle » et surtout la catastrophe de Tchernobyl (Lemarchand, 2002) auront contribué très largement à faire émerger un désir de démocratie participative en émoussant la confiance que les citoyens pouvaient placer dans les institutions censées garantir leur intégrité. La légitimité des trois «piliers » du projet moderne à l'œuvre dans la dynamique du développement industriels et technologique des cinquante dernières années que sont la Science, l'Etat et le Marché, est 
sérieusement entamée. Il apparaît finalement dans toutes ces situations que l'Etat, par l'intermédiaire de ses représentants, a failli à ses missions fondamentales de protection des citoyens, que la Science n'est plus seulement l'activité rationnelle et désintéressée par laquelle le meilleur des mondes devait advenir, mais une technoscience opératoire et sans conscience, étroitement soudée aux intérêts des lobbies industriels, et que le Marché n'est plus le rêve des libéraux anglais dans lequel une autorégulation des échanges et des passions pourrait advenir dans l'échange marchand mais un système de destruction organisé et mondialisé dépourvu de tout souci d'humanité.

Le risque n'apparait toutefois que comme une catégorie imaginaire, celle de la pensée calculante, qui domine notre époque : il est, en d'autres termes, un fait social. Le «risque » est tellement omniprésent, qu'il soit perçu dans l'autre, dans la nature, dans notre assiette, dans la jeunesse comme dans toute autre population «à risque », dans l'espace privé (protégé) comme dans le domaine public (vidéosurveillé), que nous pourrions même considérer qu'il est devenu une nouvelle catégorie de perception, une catégorie esthétique. Tout comme l'homme de la Renaissance à institué sa relation au monde sensible sous la forme du paysage, se posant en tant que sujet observateur devant l'objet-nature observé, nous pourrions dire que le risque est la relation qui s'établit désormais entre l'homme inquiet du début du XXIe siècle et un techno-monde - ou une technonature - devenus difficilement compréhensibles (Lemarchand, op. cit.). Dès lors, un rapport de méfiance semble de plus en plus caractériser les relations de la société aux technosciences (industrie nucléaire, bio et nanotechnologies, etc.) là où trente ans auparavant une confiance aveugle prônait le développement technologique comme une éthique du progrès, dans le cadre d'un pacte passé dans l'après-guerre entre l'Etat, l'industrie et le travailleur. Ce «contrat de confiance » garantissant au citoyen la protection de l'Etat et l'accroissement de son niveau de vie contre sa participation sans retenue au système - y compris dans ses loisirs, par la consommation - s'est rompu sous l'effet d'une série de «crises », qui n'en sont d'ailleurs pas tant que des effets prévisibles. Dès lors, comment la catégorie de risque, qui n'est qu'une manière parmi d'autres de dire l'incertitude, façonne-telle nos institutions, les politiques publiques, le contexte législatif ou encore les attentes de la société civile ? Depuis la publication de l'ouvrage de M. Callon et P. Lascoumes (2001), Agir dans un monde incertain, l'idée de démocratie technique, ou de démocratie participative, a fait son apparition dans le vocabulaire des gestionnaires du risque technique et industriel. S'il est irréfutable qu'un certain nombre de «nouveaux risques » a émergé dans le contexte de production d'innovations technologiques à caractère problématique (nucléaire, biotechnologies agricoles, nanotechnologies et bientôt, convergence des nano et des biotechnologies avec les technologies de l'information très investie par l'armée), la question de la régulation sociale et politique des produits de la science et de la technique n'est pas nouvelle ; elle est même inhérente à la diffusion des produits de l'activité rationnelle. C'est donc dans un contexte de nouvelle organisation des enjeux institutionnels concernant la Science, la technique, l'Etat, l'économie et la société civile que s'inscrit la présente réflexion sur le secret militaire. 
Le projet de loi $n^{\circ} 1216$ relatif à la programmation militaire pour les années 2009 à 2014 (articles 12 à 14) va accroître sans limites précises les zones d'influences protectrices du secret défense en laissant le dessin de ses contours, au coup par coup, entre les mains du gouvernement. Le voile du secret va donc s'étendre sur de grands pans de l'activité gouvernementale. Selon Dominique Barella (2009), ancien membre du Conseil Supérieur de la Magistrature, ancien président de l'Union syndicale des magistrats, dès que l'Etat estimera que cela peut gêner ses intérêts, citoyens, journalistes, magistrats, associations de défense se verront opposer à leurs questions un secret étendu : sur les risques d'une explosion, une fuite nucléaire, un accident maritime, un trafic d'arme, un fichage en réseau. Le verrouillage des secrets d'Etat grâce au secret défense pourrait donc constituer un moyen de contrôle efficace... s'il n'est pas soumis à son tour à un processus de concertation. La société civile laissera-t-elle l'Etat jeter un voile protecteur sur toutes les activités qu'il estimera lui-même secrètes ? «Ainsi, dès que de près ou de loin, un bureau, un processus informatique, un lieu de stockage, un document, une entreprise, du matériel, des produits chimiques ou nucléaires, des images, des fichiers pourront se rattacher d'une façon ou d'une autre à un supposé intérêt supérieur de la nation, tombera sur lui l'écran protecteur de l'exécutif drapé dans un intérêt d'Etat fort indéfini » poursuit Dominique Barella pour qui cette réforme crée un risque majeur pour le fonctionnement de la démocratie technique. Ainsi, dans ce projet de loi, l'incrimination des atteintes au secret de la défense nationale est modifiée par la substitution de la notion de «renseignements» à celle d' «informations» totalement indéfinie et extensible à l'infini. La conception de l'atteinte au secret de la défense nationale sera tellement étendue qu'elle se confondra avec la défense du secret d'Etat. Toute perquisition serait, de fait, rendue impossible ou totalement inefficace (article 12) obligeant le juge à révéler les raisons de sa perquisition et à décrire ce qu'il vient chercher. Dans les lieux qui abritent un secret défense (article 12 II), le juge d'instruction devra informer le président de la commission consultative du secret défense qui contrôlera les éléments saisis. Le caractère insaisissable et inutilisable dans la procédure judiciaire d'éléments classifiés qui auront été fortuitement découverts et qui n'auront pas été préalablement visés sera généralisé, même s'ils prouvent la réalisation d'un crime, par exemple de nature écologique ou industrielle.

Enfin, les populations victimes des catastrophes risquent d'être privées d'éléments importants dans les dossiers judiciaires face à un secret défense omniprésent. En cas de fuite nucléaire, d'explosion, d'accident chimique, les victimes ne pourront plus obtenir de la justice, comme à Fukushima, qu'une enquête incomplète et partiale face à un secret de la défense devenu totalement extensif. La «peur des juges » et la culture du secret ne visentelles pas les derniers remparts du droit comme garant démocratique ? Ne s'agit-il pas d'éloigner encore un peu plus les citoyens de la réalité de l'exercice du pouvoir ? Il nous revient de prendre en considération cette récente évolution programmée du droit, pour la confronter aux situations concrètes. Dans quelle direction évolueront-elles si un tel projet de loi se concrétise ? Inversement, les contextes de débat autour des installations classées secret défense peuvent-ils modifier en retour le cadre de la loi ? Le secret d'Etat est, on le voit, devenu problématique dans certaines situations alors que des situations, à l'inverse, d'extrême «transparence » semble tout autant poser problème comme nous avons pu le voir avec «l'affaire Wikileaks». 


\section{Pour conclure}

Les mutations politiques, sociales et anthropologiques de l'époque tendent d'un côté à liquider progressivement les «zones d'ombres" des instituions modernes (l'individu, la famille, la collectivité, l'Etat...) en même temps qu'elles produisent, d'un autre côté, de nouvelles forment d'opacités qui recouvrent des pans entiers des activités humaine (la recherche et le développement technologique, l'économie spéculative...). Paradis fiscaux développés au nom du secret bancaire, innovations technologiques gérées dans le plus grand secret (biotechnologies, nanotechnologies, nucléaire) au nom du secret industriel ou du secret d'Etat, autant de zones d'ombres qui font l'objet d'un appel à «plus de clarté » de la part des citoyens, en même temps que les institutions publiques (étatiques et politiques) et privées (entreprises multinationales) cherchent à protéger l'opacité d'un certain nombre de pratiques qui sont, d'une certaine manière, « inavouables » au plus grand nombre. Dès lors, tout semble se passer comme si, pour satisfaire une demande sociale de confiance, le pouvoir avait détourné le sens de la question en y répondant par des actes de transparence.

La récente « affaire Cahuzac » agit comme un puissant révélateur de ces contradictions qui ont conduit le pouvoir suprême à répondre à la situation de crise en demandant à tous les membres du gouvernement français de faire acte de transparence en publiant leur patrimoine personnel. Mais la crise en question est bien plus profonde et appelle une réflexion sur la nature des institutions démocratiques et à l'opportunité de les réformer en instituant des règles qui iraient vers plus de démocratie authentique. Face à l'incapacité du politique à gouverner véritablement, c'est-à-dire à être en prise sur les vrais problèmes (le développement, les grandes questions écologiques, la question du travail et de l'emploi, etc.) on a remplacé les problèmes sociaux sur lesquels « on ne peut rien » par des problèmes sociétaux que l'on espère pouvoir maîtriser par des décrets, c'est le sens de la demande adressées aux Ministres de déclarer leurs avoirs. Mais la véritable question posée par cette crise est bien plutôt celle de la confiance que peuvent placer les citoyens dans leurs institutions et dans ceux qui les représentent, les élus. Et la question se pose pareillement pour la science et les experts. Qu'un ministre du budget ait pu frauder est, en soi, difficilement admissible, notamment en période d'austérité. Mais plus, ce que nous révèle cette affaire tient plutôt au fait qu'après avoir délibérément menti aux citoyens, au Président et à l'Assemblée Nationale, le malheureux ait avoué sa faute. Après le parjure, cet aveu a rompu le pacte du silence qui lie la «caste » politique par le secret. Aucun des prédécesseurs de M. Cahuzac, parmi les nombreux élus qui furent jugés coupables (d'abus de bien sociaux en général) n'avait reconnu sa culpabilité. Une seule règle, en la circonstance : «n'avoue jamais !». Cette rupture du pacte est notamment à l'origine de la violence des attaques qui se sont faites jours durant plus d'un mois à l'encontre du ministre déchu. Qu'un aveu de culpabilité passe pour une trahison est à notre sens, beaucoup plus inquiétant que le fait de posséder un compte occulte, et le signe d'un grave dysfonctionnement de nos institutions politiques. 
Dès lors, on voit mal comment un surcroît de transparence pourrait permettre de mettre fin à une telle crise du politique ou à la crise des institutions, alors que la question posée est celle de la confiance que chacun est susceptible de placer dans les institutions démocratiques et en ceux qui les représentent. Or, il ne peut y avoir de confiance sans l'existence de règles qui permettent à la société d'exercer un contrôle sur le politique, sur les institutions qui la gouverne et la représente, c'est l'essence de la démocratie. La question se pose alors de savoir si les institutions de la $\mathrm{V}^{\mathrm{ème}}$ république et si notre Constitution, qui ont été bâties pour un homme, le Général De Gaulle, ne pourraient pas être repensées. C'est notamment l'intention de Yves Charles Zarca (2010) lorsqu'il propose de substituer à la légitimité de titre (le mandat donne au pouvoir l'entière liberté de faire ce qu'il veut) une légitimité d'exercice basée sur la possibilité de contrôler celui qui aura accepté de prendre une « charge », tel le Consul romain Cincinnatus retournant à sa charrue, le plaçant dans une situation d'obligation à l'égard des citoyens et non dans la posture de la seule réussite d'un plan de carrière. Dans un même registre, le philosophe Dominique Bourg tente d'ouvrir la voie à une réforme démocratique et écologique de nos institutions (Bourg, Witheside, 2010) face à la montée des périls environnementaux qu'elles sont incapables d'anticiper parce qu'elles ont été créées dans un «monde infini», le monde moderne, en questionnant la capacité des ONG et autres conférences de consensus à éclairer les choix technologiques, ou encore en proposant un nouveau Sénat et une Académie du futur. 
Arendt Hannah (2005), Le système totalitaire, Paris, Le Seuil.

Arnaud André-Jean (1993), (dir.), Dictionnaire encyclopédique de théorie et de sociologie du droit, LGDJ, Paris.

Barella, Dominique (2009), « Du secret défense à la défense des secrets d'Etat »,blog du journal Libération, [en ligne], [http://lajustice.blogs.liberation.fr/barella/2009/02/du-secretdfens.html], (février 2009)

Baudrillard Jean (1995), Le crime parfait, Paris, Galilée.

Beck Ulrich (2001), La société du risque. Sur la voie d'une autre modernité, Paris, Aubier

Berger et Luckman (1986), La construction sociale de la réalité, Paris, Méridiens Klincksieck.

Bissot, Hugues, La transparence sacrée ou le secret révélé, Droits de l'homme et dialogue interculturel [en ligne], [www.dhdi.free.fr], (juin 2013)

Bourg Dominique, Witheside Kerry (2010), Vers une démocratie écologique, le citoyen, le savant et le politique, Paris, Seuil.

Callon Michel, Lascoumes Pierre, (2001), Agir dans un monde incertain. Essai sur la démocratie technique, Paris, Le Seuil.

Clastres Pierre (1974), La Société contre l'Etat, Paris, Editions de Minuit.

Deleuze Gilles (1990), «Post-scriptum sur les sociétés de contrôle », in Pourparlers, Paris, Les éditions de Minuit.

Dupuy Jean-Pierre (2009), La marque du sacré, Paris, Fayard.

Erhenberg Alain (1998), La fatigue d'être soi, Paris, Odile Jacob.

Foucault Michel (1975), Surveiller et punir, Paris, Gallimard.

Giddens Anthony (1994), Les conséquences de la modernité, Paris, L’Harmattan.

Gueshière Peter (2000), "Sorcellerie et modernité : retour sur une étrange complicité », Politique africaine, 79, p. 17-32.

Latouche Serge (2005), L'Occidentalisation du monde, Paris, La Découverte/Poche Série "Essais", n 203.

Lemarchand Frédérick (2002), La vie contaminée, éléments pour une socio-anthropologie des sociétés épidémiques, Paris, L'Harmattan.

Marzano Michella (2009), Le Fascisme, un encombrant retour?, Paris, Larousse. 
Moreau-de-Belaing Louis (2003), L'Un sans l'autre, Paris, Anthropos.

Petitat André (1998), Secret et formes sociales, Paris, PUF.

Petitat, André (1997), " Secret et morphogenèse sociale ", Cahiers internationaux de sociologie, p. 139-160

Simmel, Georg (1991), Secret et sociétés secrètes, Circé, Paris.

Tocqueville (de), Alexis (1981), De la Démocratie en Amérique, tome II, Paris, GarnierFlammarion.

Virilio Paul (1993), L’insécurité du territoire, Paris, Galilée.

Zarca Yves Charles (2010), (sous la direction de), Repenser la démocratie, Paris, Armand Colin. 
Frédérick Lemarchand. Maitre de conférences en sociologie à l'Université de Caen, habilité à diriger des recherches. Codirecteur du Pôle Risques de la Maison de la recherche en sciences humaines de Caen. A dirigé le Laboratoire d'Analyse sociologique et anthropologique des risques. Membre du comité de rédaction de la revue Vertigo. Attaché à la compréhension des mutations profondes de l'époque contemporaine, il a mené une réflexion depuis vingt ans conjointement sur le mouvement paysan écologiste en France et sur les dynamiques du patrimoine et de la mémoire collective. Dans l'héritage de la théorie critique, il travaille depuis quinze ans sur les aspects fondamentaux des sociétés technoscientifiques (crises du nucléaire, de la vache folle, OGM, catastrophe de Tchernobyl, etc.). Il a par ailleurs enseigné huit ans à l'Université de Caen Basse-Normandie. Enseignant vacataire à l'UQAM et à l'UNIL, il s'efforce de développer des liens de coopération avec les institutions de recherche francophones sur les risques et l'environnement, en particulier au Québec. 


\section{Résumé}

Partant d'une réflexion théorique liée à une recherche sur le secret militaire, cet article tente de mettre en relation les mutations du pouvoir à l'œuvre depuis les trente dernières années et les enjeux démocratiques liés aux sociétés du risque à travers une question éthique essentielle: le statut du secret dans les démocraties. Il s'agit d'une question éthique au sens où la revendication d'un droit à la connaissance est devenue une question politique fondamentale au sein des sociétés technoscientifiques, reposant sur une apparente contradiction: la démocratie suppose une large diffusion de la connaissance, préalable à la prise de décision, alors que le pouvoir repose fondamentalement sur une logique de séparation propre au secret. Au-delà des excès du secret - qui peut recouvrir des formes de corruption - et de ceux de la transparence - essence du totalitarisme - existe-il des issues qui nous conduiraient vers une démocratie authentique?

\section{Summary}

From the starting point of a theoretical reflection related to research on military secret, this article aims at linking the ongoing mutations of power in the last 30 years and the democratic challenges linked to the societies of risk through the scope of a crucial ethical question: the status of secret in democracies. It is an ETHICAL question in the sense that advocating a right to knowledge has become a crucial political question within techno-scientific societies relying on an apparent contradiction: democracy relies on the dissemination of knowledge, prior to the taking of decisions; yet power relies heavily on a logic of separation which is specific to secrecy. Beyond the excesses of secrecy -which can encompass forms of corruption- and those of transparency -the essence of totalitarianism- are there solutions which could guide us toward an authentic form of democracy? 